\title{
Development of Neutron Polarization Measurement System for Studying $N N$ interaction in Nuclear Medium
}

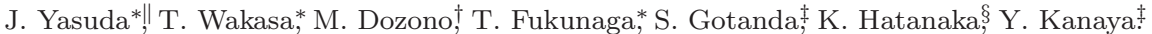 \\ Y. Maeda $\stackrel{\ddagger}{\ddagger}$. Maeda* K. Miki $\stackrel{\$}{\S}$ Y. Nishio, T. Noro, K. Ohnaka, S. Sakaguchi, ${ }^{*}$ Y. Sakemi, \\ K. Sekiguchi,**A. Tamii $\$$ T. Taguchi,**and Y. Wada** \\ *Department of Physics, Kyushu University, Higashi, Fukuoka 812-8581, Japan \\ ${ }^{\dagger}$ Center for Nuclear Study, University of Tokyo, Tokyo 113-0033, Japan \\ ${ }^{\ddagger}$ Department of Applied Physics, University of Miyazaki, Miyazaki, Miyazaki 889-2192, Japan \\ $\S$ Research Center for Nuclear Physics, Osaka University, Ibaraki, Osaka 567-0047, Japan \\ ${ }^{\top}$ Cyclotron and Radioisotope Center, Tohoku University, Sendai, Miyagi 980-8578, Japan \\ **Department of Physics, Tohoku University, Sendai, Miyagi, 980-8578, Japan \\ "yasuda@phys.kyushu-u.ac.jp \\ Published 29 February 2016
}

\begin{abstract}
We have developed the neutron polarization measurement system to perform the first polarization-transfer measurement for the exclusive $(p, n p)$ reaction. For the neutron polarization measurement, we have reconstructed the neutron polarimeter NPOL3. The NPOL3 system has been calibrated by using the polarized neutron from the ${ }^{2} \mathrm{H}(\vec{p}, \vec{n})$ reaction, and the resulting effective analyzing power is $A_{y: \text { eff }}=0.127$. For the exclusive measurement, the Large Acceptance Spectrometer (LAS) has been used for the recoil proton detection. The energy resolution of $6 \mathrm{MeV}$ is achieved for separation energy, which is sufficient to separate the $1 s$ and $1 p$ orbits for light nuclei.
\end{abstract}

Keywords: Medium effect; neutron polarization; knock-out reaction.

\section{Introduction}

Recent studies on hadron many body system are revealing that basic hadron properties would be modified in nuclear medium [1], and thus nucleon-nucleon $(N N)$ interaction might be also modified in the medium. To investigate the medium effect on $N N$ interaction, exclusive measurements of quasi-elastic knockout $(p, p N)$ reactions are one of the most direct ways since the observables are directly reflect $N N$ interaction in nuclear field. Especially, the spin observables are very useful since they are insensitive to the distortion effect.

The analyzing powers $A_{y}$ for $(p, 2 p)$ reactions have been extensively studied [24]. The data are significantly suppressed from theoretical calculations based on $N N$

This is an Open Access article published by World Scientific Publishing Company. It is distributed under the terms of the Creative Commons Attribution 3.0 (CC-BY) License. Further distribution of this work is permitted, provided the original work is properly cited. 
interaction in free space, and the suppression is a function of averaged nuclear density. There are several candidates for this $A_{y}$ suppression such as modifications of meson-nucleon coupling constants [5] and relativistic effects [6], however, the reasonable reproduction could not be achieved. Recently, polarization observables $D_{i j}$ for the same $(p, 2 p)$ reactions have been also measured. In this case, medium effects as a function of averaged nuclear density are not observed. One simple explanation is that only the relative phase of the $N N$ interaction is modified in the nuclear field since the $D_{i j}$ is insensitive to the phase while the $A_{y}$ is sensitive [7].

Recently, $A_{y}$ for $(p, n p)$ reactions have been measured for ${ }^{2} \mathrm{H},{ }^{6} \mathrm{Li}$, and ${ }^{12} \mathrm{C}$ targets [8]. In this case, the data are well reproduced by the theoretical calculations. It suggests that the medium effects are different in p-p and p-n channels. In order to investigate this possible isospin dependence of the nuclear medium effects, the $D_{i j}$ data for the $(p, n p)$ reaction are highly desired.

In the present study, we developed the $D_{N N}$ measurement system for the $(p, n p)$ reaction. For the neutron polarization measurement, we have reconstructed the neutron polarimeter NPOL3 to reduce the back ground. By using the polarized neutron from the ${ }^{2} \mathrm{H}(\vec{p}, \vec{n})$ reaction, we have calibrated the NPOL3 system. For the exclusive $(p, n p)$ measurement, we have used the Large Acceptance Spectrometer (LAS) to detect the recoil proton. We performed the test experiment for the exclusive ${ }^{2} \mathrm{H}(p, n p)$ measurement to investigate the performance of the present system.

\section{Experimental Setup}

Figure 1 shows a schematic view of the experimental setup at the Research Center for Nuclear Physics (RCNP), Osaka University. Polarized protons were produced in the HIPIS [9] and were accelerated up to $T_{p}=53$ and $296 \mathrm{MeV}$ using the azimuthally varying field (AVF) and ring cyclotrons, respectively. The polarization axis of the beam was normal and the beam polarization was monitored using the beam line polarimeter. A typical beam polarization was $p_{N} \sim 0.4$. The beam was bombarded on the deuterated polyethylene $\left(\mathrm{CD}_{2}\right)$ target with a thickness of $16.7 \mathrm{mg} / \mathrm{cm}^{2}$. The outgoing neutrons were measured by the neutron polarimeter NPOL3 [10] at $\theta_{\mathrm{NPOL}}=22^{\circ}$ with flight-pass length $20 \mathrm{~m}$. The recoil protons were also measured by the LAS spectrometer at $\theta_{\mathrm{LAS}}=65.0^{\circ}$ to satisfy the condition for the recoil momentum $p_{3} \simeq 0$.

\section{Neutron Polarization Measurement}

The NPOL3 system [10] has two main neutron detector planes, hodoscopes (HD1 and HD2). Each plane consists of 10-stacked one-dimensional position sensitive plastic scintillator (BC408) with a size of $100 \times 10 \times 5 \mathrm{~cm}^{3}$ (see Fig. 1). The incident neutron energy is determined by the time-of-flight (TOF) to a given hodoscope. The thin plastic scintillator plane CPV is used to identify charged particles. In the polarimetry mode of NPOL3, the neutron polarization is analyzed by using the $\vec{n}+p$ scattering in a given hodoscope, and the recoil proton is detected in the 


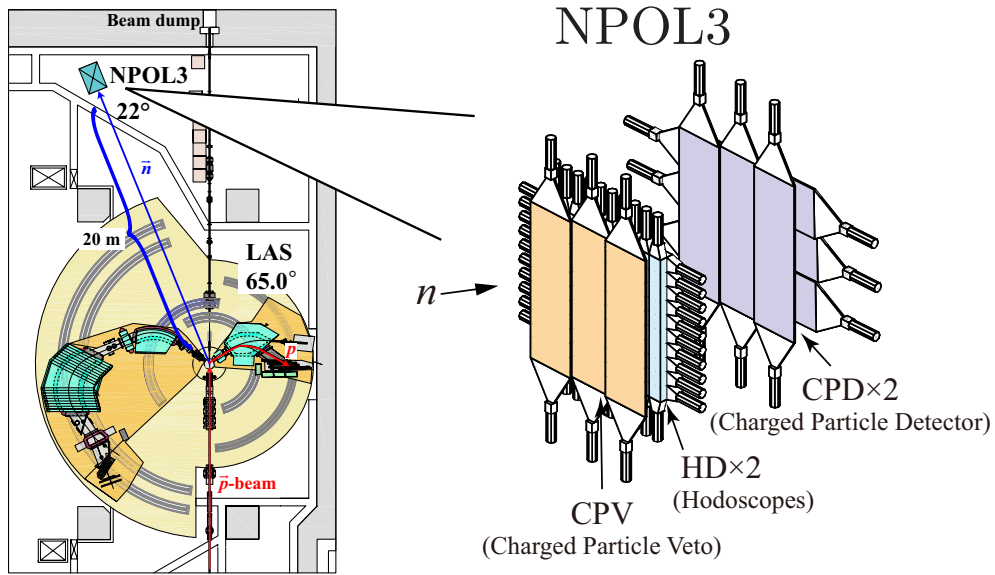

Fig. 1. A schematic view of experimental setup. The polarized proton beam was accelerated by the AVF and ring cyclotrons up to $296 \mathrm{MeV}$ and bombarded on ${ }^{2} \mathrm{H}$ target. The outgoing neutrons were measured by the neutron polarimeter NPOL3 and the recoil protons were also measured by the LAS spectrometer.

last plane of NPOL3. The last plane of the original NPOL3 has a large volume of $100 \times 100 \times 10 \mathrm{~cm}^{3}$. In the $(p, n p)$ setup, the counting rate would be extremely high due to the background from the beam duct and the beam dump. Therefore, we have replaced the last plane for detecting recoil proton by two planes of charged particle detectors (CPDs) as shown in Fig. 1. Each CPD is made of three sets of one-dimensional position sensitive plastic scintillators (BC408) with a size of $102 \times 35 \times 0.5 \mathrm{~cm}^{3}$. By using the position information from the two-planes, we can obtain the two-dimensional position of recoil proton.

The effective analyzing power $A_{y \text {; eff }}$ of NPOL3 was determined by using the polarized neutron from the ${ }^{2} \mathrm{H}(\vec{p}, \vec{n})$ reaction at $T_{p}=296 \mathrm{MeV}$. The polarization transfer $D_{N N}$ for ${ }^{2} \mathrm{H}(\vec{p}, \vec{n})$ at $T_{p}=346 \mathrm{MeV}$ was precisely measured and the results were well reproduced by plane-wave-approximation (PWIA) calculations $[11,12]$. Therefore, we can obtain the reliable $D_{N N}^{\text {theor }}$ value at $T_{p}=296 \mathrm{MeV}$ based on the PWIA calculation. The experimentally obtained $D_{N N}^{\exp }$ is given by

$$
D_{N N}^{\exp }=\frac{1}{p_{N}}\left(\frac{\epsilon\left(1+p_{N} A_{y}\right)}{A_{y: \text { eff }}}-P\right),
$$

where $\epsilon, p_{N}$, and $P$ are the double scattering asymmetry, the polarization of incident proton, and the induced polarization, respectively. Here, we assumed the induced polarization $P=0$ since $D_{N N}$ is insensitive to $P$.

The $A_{y \text {;eff }}$ value is determined so that the experimental and theoretical $D_{N N}$ values become consistent. Figure 3 shows the results of the experimental $D_{N N}^{\exp }$ in which the $A_{y \text {; eff }}$ has been adjusted to reproduce the theoretical $D_{N N}^{\text {theor }}$ in the energy

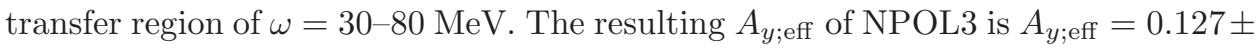




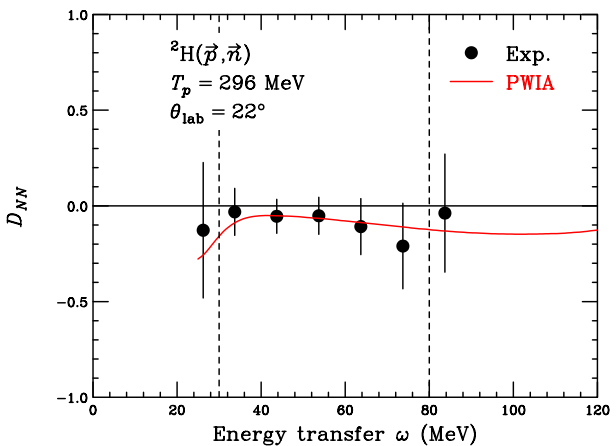

Fig. 2. Polarization transfer $D_{N N}$ for the ${ }^{2} \mathrm{H}(\vec{p}, \vec{n})$ reaction at $T_{p}=296 \mathrm{MeV}$. The red solid line indicates the PWIA prediction.

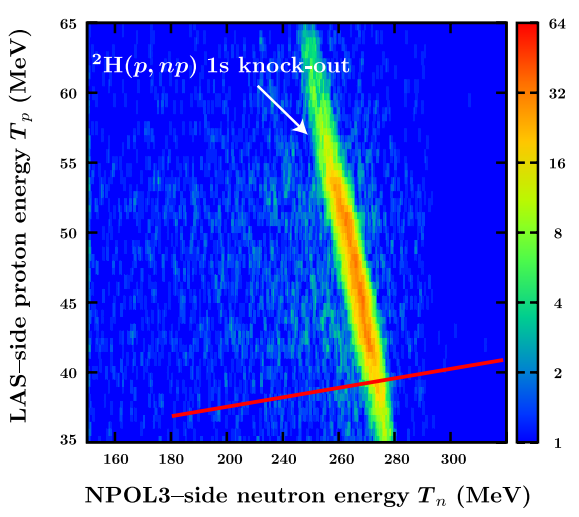

Fig. 4. A two-dimensional energy spectrum between proton and neutron energies for the ${ }^{2} \mathrm{H}(p, n p)$ reaction. By projecting to the red line, we can obtain the separation energy spectrum.

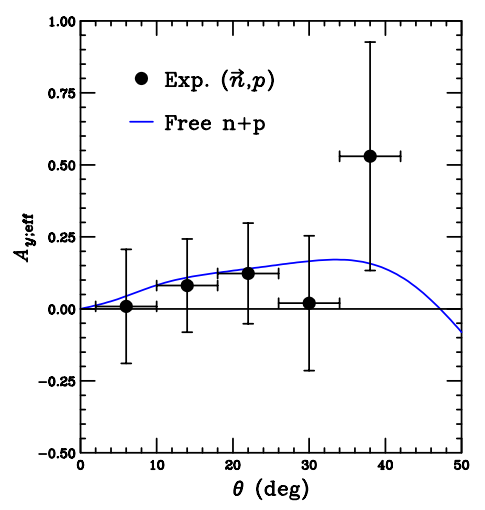

Fig. 3. Angular distribution of the effective analyzing powers of NPOL3. The solid curve represents the $A_{y}$ for the free $\vec{n}+p$ scattering.

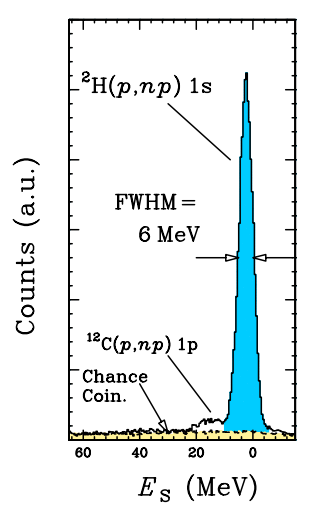

Fig. 5. A separation energy spectrum for the ${ }^{2} \mathrm{H}(p, n p)$ reaction.

0.059, where the uncertainty includes systematic and statistical contributions. The small $P$ dependence of $D_{N N}$ was investigated and its effect is also taken into account as the systematic uncertainty.

Figure 4 shows the effective analyzing power as a function of double scattering angle. The solid line shows the analyzing power $A_{y}$ for the free $n+p$ scattering [13]. The measured angular distribution is very similar to that of the $n+p$ scattering, supporting the proper kinematical selection of the $\vec{n}+p$ events in the present analysis.

The performance of a polarimeter can be measured by its figure-of-merit (FOM) value defined as FOM $=\varepsilon_{\text {D.S. }}\left(A_{y ; \text { eff }}\right)^{2}$, where $\varepsilon_{\text {D.S. }}$ is the double scattering efficiency. The $\varepsilon_{\text {D.S. }}$ value of NPOL3 is $0.42 \pm 0.04 \%$. Table 1 compares the FOM value of the 
Table 1. Comparison of the FOM values of present NPOL3, original NPOL3 [10], and INPOL [14].

\begin{tabular}{c|ccc}
\hline \hline & the present NPOL3 & the original NPOL3[10] & INPOL[14] \\
\hline FOM & $0.67 \times 10^{-4}$ & $0.89 \times 10^{-4}$ & $0.81 \times 10^{-4}$ \\
\hline \hline
\end{tabular}

present NPOL3 system with those of the original NPOL3 and INPOL [14] systems. A small difference of the FOM values would be due to the different neutron energies and detector geometries.

\section{Exclusive Measurement}

The momentum of the recoil proton was analyzed with the Large Acceptance Spectrometer (LAS) which consists of a quadrupole magnet and a dipole magnet. At the focal plane of the LAS, a one-dimensional position sensitive plastic scintillator with a size of $195 \times 15 \times 0.5 \mathrm{~cm}^{2}$ was used for position reconstruction. We can evaluate the separation energy $E_{s}$ for the $(p, n p)$ reaction by using the neutron momentum information from the NPOL3,

Figure 4 shows the two-dimensional energy spectrum between proton and neutron energies for the ${ }^{2} \mathrm{H}(p, n p)$ reaction at $T_{p}=296 \mathrm{MeV}$. The locus is clearly seen in the spectrum. The separation energy spectrum can be obtained by projecting to the red-line, and the result is shown in Fig. 5. The peak observed at $E_{S} \sim 0$ corresponds to the ${ }^{2} \mathrm{H}(p, n p) 1 s$ knocked-out reaction. The yellow shaded area represents the chance coincidence events evaluated by using the neutron and proton TOF difference, and a low background level has been achieved. The energy resolution is $\Delta E_{S} \simeq 6 \mathrm{MeV}$ in FWHM, which is sufficient to separate $1 s$ and $1 p$ orbits for light nuclei.

\section{Summary}

We have developed the neutron polarization measurement system for the $(p, n p)$ reaction with the following two steps; neutron polarization measurement and the exclusive measurement. For the neutron polarization measurement, the neutron polarimeter NPOL3 has been reconstructed to reduce the backgrounds. We have calibrated the modified NPOL3 by using polarized neutrons from the ${ }^{2} \mathrm{H}(p, n)$ reaction. The effective analyzing power and the double scattering efficiency are $A_{y \text {;eff }}$ $=0.127$ and $\varepsilon_{\text {D.S. }}=0.42$, respectively. This performance is almost similar to those of the original NPOL3 and INPOL systems. For the exclusive measurement, the Large Acceptance Spectrometer (LAS) was used for recoil proton detection. We have achieved the energy resolution of $6 \mathrm{MeV}$ in FWHM which is sufficient to separate $1 s$ and $1 p$ orbits for light nuclei.

\section{References}

1. T. Hatsuda and S. H. Lee, Phys. Rev. C 46, R34 (1992). 
2. K. Hatanaka et al., Phys. Rev. Lett. 78, 1014 (1997).

3. T. Noro et al., Phys. Rev. C 72, 041602R (2005).

4. T. Noro et al., Phys. Rev. C 77, 044604 (2008).

5. T. Noro et al., Nucl. Phys. A 629, 324c (1998).

6. T. Noro et al., Nucl. Phys. A 633, 517c (2000).

7. J. M. Moss, Phys. Rev. C 26, 727 (1982).

8. Y. Yamada, PhD thesis, Kyushu University, 2010.

9. K. Hatanaka, K. Takahisa, H. Tamura, M. Sato, and I. Miura, Nucl. Instrum. Methods Phys. Res. A 384, 575 (1997)

10. T. Wakasa et al., Nucl. Instrum. Methods Phys. Res. A 547, 569 (2005).

11. T. Wakasa et al., Phys. Rev. C 59, 3177 (1999).

12. T. Wakasa et al., Phys. Rev. C 69, 044602 (2004).

13. R. A. Arndt and L. D. Roper, Scattering Analyses Interactive Dial-in (SAID) program, phase shift solution SM89, Virginia Polytechnic Institute and State University (unpublished).

14. M. Palarczyk et al., Nucl. Instr. and Meth. Phys. Res. A 457 (2001) 309. 